\title{
Photocatalytic Benzylic Fluorination
}
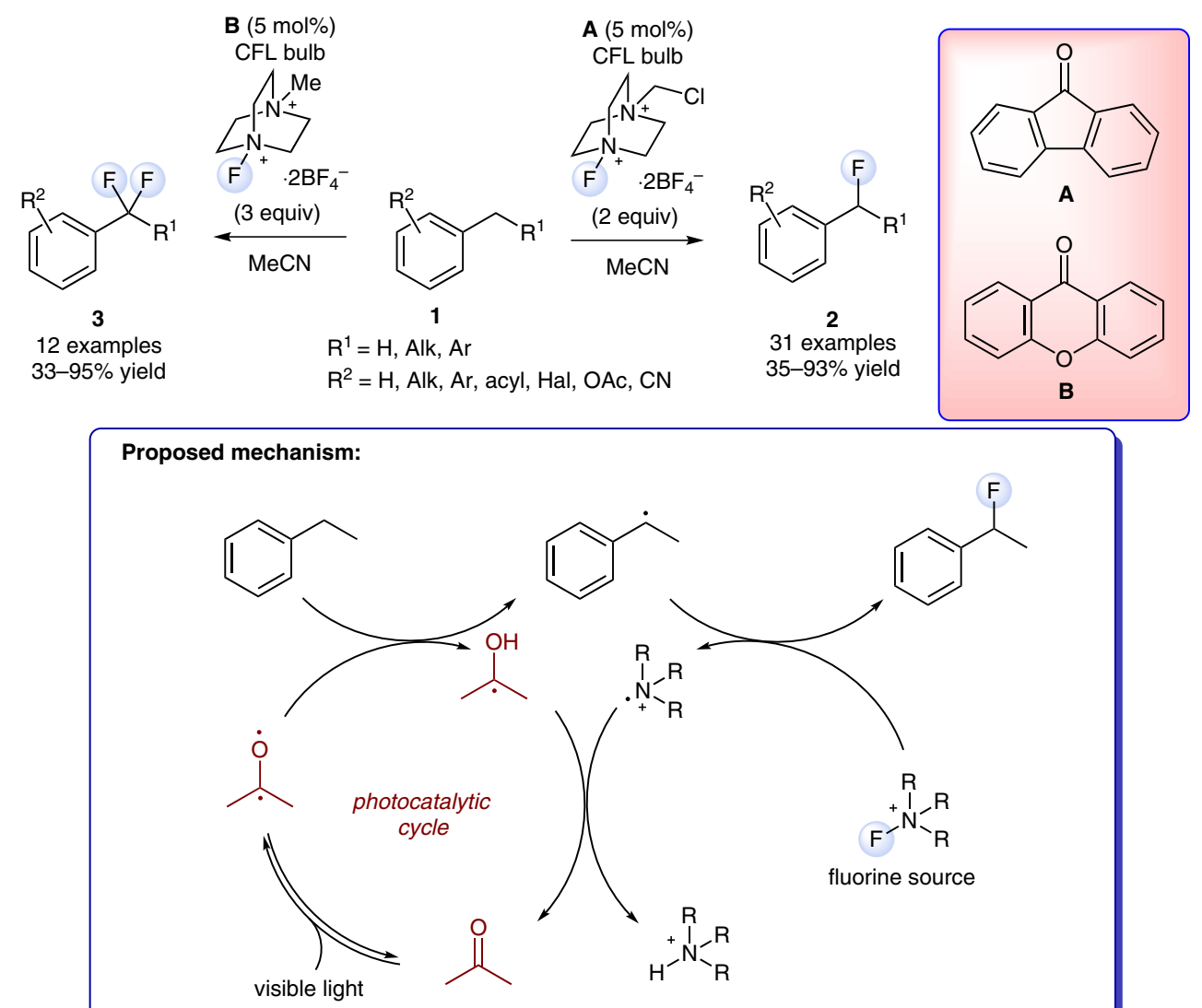

\section{Key words}

fluorination

visible-light catalysis

ketone catalysis
Significance: A photocatalytic benzylic fluorination is reported by Chen and co-workers. Promoted by visible light, the photoexcitation of the ketone organocatalyst generates a short-lived diradical species, which abstracts a benzylic hydrogen from the starting material. Subsequently, the fluorine source delivers the F-atom and regenerates the catalyst. The methodology is operationally convenient and converts a large variety of substrates into the corresponding mono- and difluorinated products using a simple compact fluorescent light (CFL) bulb and commercially available Selectfluor (A) and Selectfluor II (B).
Comment: In the last few years, the interest of the scientific community in the synthesis of fluorinated compounds has risen impressively due to the importance of these substances in pharmaceutical and material sciences. Therefore, the development of selective and mild procedures for the introduction of fluorine atoms, even in a racemic fashion, is very attractive. Here, the authors present a solid protocol to achieve this target. A wide variety of substrates was reacted to give the fluorinated products in good to excellent yields, highlighting the efficiency of the disclosed photocatalytic system.

SYNFACTS Contributors: Benjamin List, Mattia Riccardo Monaco 